\title{
Prevalence, characteristics and risk factors of imported and local malaria cases in North-Western Province, Zambia: a cross-sectional study
}

\author{
Maureen N. Chipoya and Nzooma M. Shimaponda-Mataa* (1)
}

\begin{abstract}
Background: Imported malaria is a major challenge for countries that are in malaria elimination stage such as Zambia. Legitimate cross-border activities add to the risk of transmission, necessitating determination of prevalence, characteristics and risk factors of imported and local malaria.

Methods: This cross-sectional study was conducted in 103 consented child and adult patients with clinical malaria symptoms, from selected health facilities in north-western Zambia. Patient demographic data and blood samples for malaria microscopy and full blood count were obtained. Chi-square and penalized logistic regression were performed to describe the characteristics and assess the risk factors of imported and local malaria in North-Western Province.

Results: Overall, malaria prevalence was $78.6 \%$ with $93.8 \%$ Plasmodium falciparum and $6.2 \%$ other species. The local cases were 72 (88.9\%) while the imported were $9(11.1 \%)$ out of the 81 positive participants. About $98.6 \%$ of the local cases were P. falciparum compared to $55.6 \%\left(X^{2}=52.4 ; p<0.01\right)$ P. falciparum among the imported cases. Among the imported cases, $44 \%$ were species other than P. falciparum $\left(x^{2}=48 ; p<0.01\right)$ while among the local cases only $1.4 \%$ were. Gametocytes were present in $44 \%$ of the imported malaria cases and only in $2.8 \%$ of the local cases $\left(x^{2}=48\right.$; $p<0.01$ ). About $48.6 \%$ of local participants had severe anaemia compared to $33.3 \%$ of participants from the two neighbouring countries who had $\left(x^{2}=4.9 ; p=0.03\right)$. In the final model, only country of residence related positively to presence of species other than P. falciparum $(\mathrm{OR}=39.0, \mathrm{Cl}[5.9,445.9] ; \mathrm{p}<0.01)$ and presence of gametocytes $(\mathrm{OR}=23.1, \mathrm{Cl}[4.2,161.6] ; \mathrm{p}<0.01)$.

Conclusion: Malaria prevalence in North-Western Province is high, with P. falciparum as the predominant species although importation of Plasmodium ovale and Plasmodium malariae is happening as well. Country of residence of patients is a major risk factor for malaria species and gametocyte presence. The need for enhanced malaria control with specific focus on border controls to detect and treat, for specific diagnosis and treatment according to species obtaining, for further research in the role of species and gametocytaemia in imported malaria, cannot be overemphasized.
\end{abstract}

Keywords: Local malaria, Imported malaria, Risk factors, Gametocytaemia, Zambia

*Correspondence: nzomataa@gmail.com

Department of Biomedical Sciences, School of Health Sciences, University of Zambia, Ridgeway Campus, Lusaka, Zambia

\section{Background}

Malaria continues to be among the most prevalent parasitic diseases experienced worldwide [1]. Globally, an estimated 228 million malaria cases and 405,000

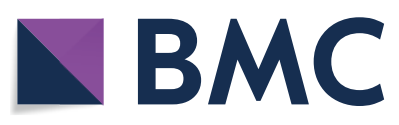

(c) The Author(s) 2020. This article is licensed under a Creative Commons Attribution 4.0 International License, which permits use, sharing, adaptation, distribution and reproduction in any medium or format, as long as you give appropriate credit to the original author(s) and the source, provide a link to the Creative Commons licence, and indicate if changes were made. The images or other third party material in this article are included in the article's Creative Commons licence, unless indicated otherwise in a credit line to the material. If material is not included in the article's Creative Commons licence and your intended use is not permitted by statutory regulation or exceeds the permitted use, you will need to obtain permission directly from the copyright holder. To view a copy of this licence, visit http://creativecommons.org/licenses/by/4.0/. The Creative Commons Public Domain Dedication waiver (http://creativecommons.org/publicdomain/zero/1.0/) applies to the data made available in this article, unless otherwise stated in a credit line to the data. 
deaths were recorded in 2018 compared to 231 million cases and 416,000 deaths in 2017 [2]. The majority of this burden rests on sub-Saharan Africa and India, which carry almost $85 \%$ of it [2]. In the African Region, malaria accounts for about $93 \%$ of the cases [2]. In Zambia, malaria is equally of concern as it is a major cause of mortality and morbidity [3] in highly endemic areas [46]. As of 2018, the Ministry of Health reported presumed and confirmed cases of up to 5,193,723 and deaths of up to 1209 [2].

The malaria burden in Zambia is greatly influenced by climatic factors, such as rainfall and temperature [7]. Rainfall mainly contributes to the availability of breeding habitat for mosquito vectors [8]. On the other hand, temperature determines the length of development of larval mosquitoes and malaria parasites, as well as the lifespan and the rate of blood feeding of adult vectors [8]. The burden of malaria continues to be highest in Northern, Luapula and Muchinga provinces, which fall in the high malaria transmission zone [6]. These are followed by other provinces including the North-Western Province $[9,10]$, which is officially known to belong to the medium malaria transmission zone [6]. North-Western Province has high rainfall, many rivers, thick forestry, dams and swamps. Further, the major mining activities in the province allow for various occupational activities, leading to high population [11]. The suitable environmental conditions would speak to the generally high malaria cases [7, 8].

Focussing more on North-Western Province, this is a province bordered by two countries, the Democratic Republic of Congo and Angola [11]. This geographic location may contribute to malaria importation given that malaria parasites do not respect national borders and are exacerbated by human mobility [12]. The Democratic Republic of Congo and Angola were among the six leading contributors to the $85 \%$ global malaria mortality burden that was concentrated in 20 countries in the African region and India in 2018 [2]. In 2018, the African sub-region had about 49 million estimated cases representing a 7\% increase to which the Democratic Republic of Congo and Angola were the leading contributors by approximately $55 \%$ and $14 \%$, respectively [2].

Among the five species of malaria, Plasmodium falciparum accounted for $99.7 \%$ of morbidity in the World Health Organization (WHO) African Region in 2018 [2]. Although the WHO shows only $2 \%$ Plasmodium vivax and $98 \%$ P. falciparum in Africa, and only P. falciparum in southern Africa [2], Sitali and others showed a Plasmodium species distribution of $88.4 \%$ P. falciparum, 0.8\% Plasmodium malariae and 0.4\% Plasmodium ovale in Luapula and Eastern provinces of Zambia [13]. Plasmodium vivax was also reported but only as mixed infections with other species and these were distributed as $0.2 \%$ P. falciparum with $P$. vivax and $0.2 \%$ P. falciparum with $P$. malariae and $P$. vivax [13]. The rest were mixed infections of $6.5 \%$ P. falciparum with P. malariae, $2.1 \%$ $P$. falciparum with $P$. ovale and $1.3 \%$ P. falciparum with $P$. malariae and $P$. ovale [13]. Zhou et al. also showed importation of $P$. falciparum, $P$. ovale and $P$. vivax to China from 20 African countries, with Angola, Congo, Equatorial Guinea, Nigeria, and Liberia being the top five sources of $P$. ovale and only Angola, Equatorial Guinea, Congo, and Guinea being sources of P. vivax [14]. Additionally, according to unpublished hospital records from some health facilities in North-Western Province, the species being seen in the province may include species other than P. falciparum. Considering that most Government Health Facilities use the $P$. falciparum specific rapid diagnostic tests (RDTs), such species are likely to be missed in some health facilities that have no laboratory capacity to detect other species.

Reducing the incidence of malaria is a national priority that requires a focused comprehensive and consistent approach in order to achieve the vision of "a malaria free-Zambia by 2030" [15]. So far, the National Malaria Elimination Centre (NMEC) efforts have contributed to a $40 \%$ reduction of deaths and a promising $40 \%$ reduction in cases by 2020 [2]. Although Zambia is not among the 21 countries that are part of the WHO "E-2020 initiative" identified by the WHO to work towards the 2020 elimination milestone of the global malaria strategy [2], it is a member of the Elimination Eight (E8) Regional Initiative, a similar elimination initiative at regional level in Southern Africa [16]. This initiative includes eight countries and works in a phased approach to eliminate malaria initially in four frontline countries i.e. Botswana, Namibia, South Africa and Swaziland by 2020, and later pave the way for elimination in four second-line countries (i.e. Angola, Mozambique, Zambia and Zimbabwe) by 2030 [16]. In supporting the four frontline countries, Zambia and the other three second-line countries are working to scale up malaria control, logistically, strategically and politically especially in border areas, in terms of a series of cross-border initiatives [16]. This is because increasing travel to endemic areas in recent decades contributes to imported malaria cases, which continue to pose challenges for diagnosis and management [16]. This is especially so, given the fact that currently, criteria on definitions of time periods qualifying a case to be termed an import, are not clear or standardized [17]. The criteria vary from 18 days to 2 months among countries and 3 months by the WHO [17]. Data on the features of imported cases can provide valuable information about both the epidemiology of malaria in endemic regions where surveillance systems are weak [12] as well 
as responsible factors in non-endemic regions [18]. The movement of malaria in endemic countries has also contributed to the spread of drug resistance and threatens long-term eradication goals $[15,19]$.

Control and treatment strategies in countries like Zambia, that are in the elimination stage should account for the continued globalization of malaria and increase efforts to understand patterns of human and malaria parasite movement $[8,12]$. Further, how such information can guide control and elimination strategies especially given the increasing focus on malaria importation and the vulnerability of countries to resurgence should also be prioritized $[8,12]$. As such, studying the characteristics of the local and imported malaria cases prevailing will assist in the development of more effective future malaria surveillance and prevention programmes [8], accurate detection and diagnosis [20], streamlined treatment depending on species and the varying severity of malaria and in educating the community in adherence to preventive measures. This study, therefore, sought to evaluate the characteristics of imported and local malaria cases in North-Western Province, Zambia. It further sought to determine the prevalence of malaria and highlight risk factors that could be considered in targeted prevention and control strategies.

\section{Methods}

\section{Study design}

A health facility based, cross sectional study was conducted from March to September 2019. Participants were drawn from patients of all age groups from 3 months old, with signs and symptoms of malaria and seeking medical attention from the three selected health facilities of North-Western province, Zambia.

\section{Study setting}

The study was conducted in North-Western Province, one of the ten Provinces of Zambia. This province covers an area of $125,826 \mathrm{~km}^{2}$, has a population of 727,044 and a population density of 5.80 per $\mathrm{km}^{2}$ [21]. It is one of the sparsely populated provinces in the country. The province is divided into ten districts namely, Chavuma, Ikelenge, Kabompo, Kasempa, Manyinga, Mufumbwe, Mwinilunga, Solwezi, Mushindamo and Zambezi. The rural population constitutes $77.5 \%$, while the urban population makes up the remaining $22.5 \%$. North-Western Province is bordered by Angola in the west, the Democratic Republic of Congo (Congo D. R.) in the north, Copperbelt Province in the southeast, Central Province in the south, Western Province in the west [11]. The major activities that take place within the province are farming, fishing, trading and mining. The study sampled from three health centres nearest to Congo and
Angola namely; Jimbe Rural Health Centre, $2 \mathrm{~km}$ away from Angola and about $390 \mathrm{~km}$ away from Solwezi District; Sachibondu Rural Health Centre, $16 \mathrm{~km}$ away from Congo and about $321 \mathrm{~km}$ away from Solwezi District.; and Mushindamo Rural Health Centre, in Mushindano District, $15 \mathrm{~km}$ away from Congo and about $137 \mathrm{~km}$ from Solwezi District.

Both Jimbe and Sachibondu Rural Health Centres are in Ikelenge District which is located $340 \mathrm{~km}$ from Solwezi District. Jimbe Rural Health Centre has an inpatient capacity of five beds, and covers a population of 3384 people. Sachibondu Rural Health Centre has an in-patient capacity of 25 beds. It covers a population of about 5000 people. Mushindano Rural Health Centre has an in-patient capacity of 16 beds and covers a population of about 9200 people.

Jimbe and Mushindano Rural Health Centres have no Laboratories and referral cases are mostly sent to Solwezi General Hospital.

\section{Inclusion and exclusion criteria}

Inclusion criteria for the study were: attendance at any of the three selected health facilities, of all age groups starting from 3 months old, with signs and symptoms of malaria, residence in North-Western Province and no history of travel 4 weeks prior to attending the health facility, residence in Angola or Congo with no history of travel 4 weeks prior to attending the health facility apart from travel to health facility which should have taken place within 5 to 7 days of leaving their residence.

Patients were excluded from the study if they: were under 3 months of age, were extremely ill and in need of urgent medical attention at the time of testing, had any known complications from diseases other than malaria, had taken any anti-malarial drug in the previous 3 weeks.

Travel history and anti-malarial drug data were obtained via open-ended questions asking specifically when they had last travelled out of their district or crossed borders or taken anti-malaria drugs. This was done in order to mask the intent of the questions and reduce bias. The study also used calendars to minimize recall bias by allowing the recall process to be as independent as possible.

\section{Sample size determination and enrolment}

An estimated sample size of 103 was obtained based on the $6 \%$ prevalence of malaria in the medium malaria transmission zone where North-Western Province falls [22]; by sample size formula for descriptive studies, $\mathrm{N}=\mathrm{Z}^{2} \mathrm{p}(100-\mathrm{p}) / \boldsymbol{e}^{2}$; where $\mathrm{N}(87)$ was calculated as the sample required; $Z$, the Standard error (1.96); e, Precision required $(95 \%, 0.05)$ and p, Estimated prevalence (6\%) [22]; and after factoring in non-response rate of $15 \%$, 
implying a response rate of $85 \%$; the adjusted sample size was calculated as $87 /(1-0.15)=102.7$; Sample size $(\mathrm{n})=103$. As such, a total of 103 consented and qualifying patients, were enrolled by convenience sampling. These were broken down as follows: Jimbe-34 patients; Sachibondu -34 patients; and Mushindamo-35 patients.

\section{Data collection}

All data collection was performed using standard recruitment and testing protocols by specific trained study staff to reduce bias. After confirming that the patients had not travelled and had not taken any anti-malarial drug as per inclusion and exclusion criteria, patients were taken through the informed consent (and or assent). This process was to furnish them with information about the study and request them to voluntarily participate in the study. Adult patients were requested to sign or fingerprint on the consent form while for child patients, parents or guardians assented by the same token.

For all consented (assented) patients, clinical data including demographic details of the patients, risk factors, and presenting signs and symptoms were collected from medical records of the patients after clinical examinations by the Health Professionals Council of Zambia registered Clinicians in the facilities; and through semistructured interviews with patients, parents or guardians by the study team. The interviews, study information sheet and written informed consent were developed in English but administered to the patients in a specific local language the patients or guardians were comfortable with among the four main languages namely; Lunda, Luvale, Kaonde and Lamba spoken in North-Western Province, and Lunda and Swahili for participants from Angola and Congo, respectively.

Finger prick blood specimens for RDTs and venous blood in EDTA containers for thick and thin blood smears as well as full blood count (FBC) determination, were obtained from the recruited patients.

\section{Description of laboratory procedures}

The SD BIOLINE Malaria Ag P.f POCT, a rapid qualitative diagnostic test manufactured by Standard Diagnostics, Inc., used for the detection of histidine-rich protein II (HRP-II), a malaria antigen specific to P. falciparum in human blood specimens, was employed. This was mainly for initial determination of $P$. falciparum positive individuals to facilitate immediate treatment needs. Plasmodium falciparum positive result was confirmed by the appearance of two red/pink lines in the test area of the RDT cassette representing the test and control reactions while a negative result was confirmed by the appearance of only one red/pink control line. This process was conducted for all the 103 participants enrolled and results were recorded as SD BIOLINE Malaria Ag P.f POCT RDT positive or negative.

From the venous blood specimens collected, both thick and thin films were prepared and the haemoglobin (HB) levels determined. For the samples collected from Jimbe and Sachibondu Health centres, HB determination and smear preparations were done at Mwinilunga General Hospital by a laboratory technician, while for those collected from Mushindamo Health centre, both procedures were performed at Solwezi General Hospital by laboratory technicians stationed there.

All slides from the three health facilities were read at Solwezi General Hospital by the principal investigator as the first reader. The second and third (blind) slide readers were a Medical Laboratory Technologist and a Biomedical Scientist to confirm species and parasitaemia for quality control purposes, to reduce bias.

\section{Determination of parasitaemia and species by microscopy}

Thin blood smears were air dried, fixed with $95 \%$ methanol for $1 \mathrm{~min}$ and then stained in three percent Giemsa for $45 \mathrm{~min}$, while thick blood smears were dehaemoglobinized in buffer for 30-60 s for transparency of the red blood cells then stained with ten percent Giemsa at $\mathrm{pH}$ 7.2-7.4 for $15 \mathrm{~min}$. The stained smears were examined under light microscopy at $100 \times$ magnification. Thick blood smears were used for detection and quantification both asexual and sexual forms of malaria parasites. Thin blood smears were used for species identification $[9,23$, 24].

Peripheral parasite density calculations were based on the number of asexual forms and leukocytes or white blood cells (WBCs) counted [23] and converted into parasites per microlitre of blood based on the WHOrecommended average of 8000 WBCs per microlitre of blood in every human being [25]. Briefly, this procedure involved tallying the parasites (using one tally counter) against WBCs (using a second tally counter), until the slide reader counted 100 parasites or $200 \mathrm{WBCs}$, whichever came first. Thereafter, expressing the results as parasites per microlitre of blood, using the formula: Parasites/ microlitre blood $=($ parasites $/ \mathrm{WBCs}$ counted $) \times 8000$ WBCs per microlitre blood. Where no parasites were detected, slide readers examined up to $500 \mathrm{WBCs}$ or 200 microscope fields of the thick films before assigning a negative result.

\section{Evaluating the severity of malaria in imported and local malaria cases}

Given that severe malaria is defined as either Blantyre coma score $<5$ or severe anaemia $<5 \mathrm{~g} / \mathrm{dl}$ when they co-exist with parasitaemia and an auxiliary temperature of $>37.5^{\circ} \mathrm{C}$ and other criteria as listed in the WHO 
criteria for severe malaria; and uncomplicated malaria is defined as $P$. falciparum parasitaemia and an axillary temperature of $<37.5{ }^{\circ} \mathrm{C}$, or parasitaemia and symptoms suggestive of malaria [26-28], severity of anaemia was determined based on haemoglobin levels in the participants.

HB levels were determined using full blood counts obtained from venous blood samples that were run on ABX micros 60 FBC machine. Participants who had haemoglobin levels below $5.0 \mathrm{~g} / \mathrm{dl}$ [26-28] were considered as having severe anaemia, while participants with haemoglobin levels higher than $5.0 \mathrm{~g} / \mathrm{dl}$ were considered to have mild or moderate anaemia.

\section{Determining risk factors associated with imported and local malaria cases}

The following data i.e. age, gender and country of residence were analysed statistically to determine the risk factors associated with the outcomes. Data analysis staff were blinded to patient exposure and outcome status in order to reduce bias. Data entry and cleaning were done concurrently with laboratory procedures. This allowed for re-confirmation with patient records or deleting and replacing of potential participants with completely missing data.

\section{Statistical analysis}

The data was analyzed using Microsoft Excel 2013, IBMStatistical Package for Social Sciences (IBM-SPSS) version 23 and $\mathrm{R}$ version 4.02. In the univariate analysis, data were described by summarizing continuous variables such as age, into means and standard deviations and reporting categorical variables such as gender, malaria positivity, anaemia and malaria species as frequencies and percentages.

Chi-Square $\left(x^{2}\right)$ was used for the bivariate analysis to determine relationships between categorical, independent variables i.e. country, age and gender with the outcome variables malaria positivity, malaria species, severity and gametocyte presence.

Given the following considerations (i) that species other than $P$. falciparum are uncommon in patients attending most health facilities in Zambia, (ii) that gametocyte detection by microscopy is usually low; (iii) that some outcome categories in the data set were so small and highly unbalanced, it was recognized that this would cause an underestimation of the probability of occurrence and biased standard errors [29]. Therefore, Firth logistic regression method, a type of penalized likelihood models, which is recommended for rare events and also deals with quasi-complete separation of data points to reduce bias, was used [30]. Two single-predictor Firth logistic regression models were run in $\mathrm{R}$ version 4.02 in the Windows 10 environment, using the "logisticf" package [31], with results interpreted the same way as in traditional logistic regressions. The models were run with presence of (a) species and (b) gametocytes as dependent, outcome variables and country, gender and age as predictors and independent variables. Both outcome and predictor variables, were coded with one (1) when the value of interest was present and with zero (0) for a value otherwise. Ultimately the two models were used to estimate country-associated risks for outcome or dependent binary variables, malaria species and gametocyte presence while controlling for confounders, such as age and gender. Odds ratios and 95\% confidence intervals (CI) were reported. Statistical significance was set at a level of $\mathrm{p}<0.05$.

\section{Results}

\section{Demographic characteristics}

Out of the 103 participants, 43 (41.7\%) were males and $60(58.3 \%)$ females, with a mean age of 12 years [range: 0.3 (4 months)-72 years; standard deviation: 11.7]. Table 1 presents a summary of the demographic characteristics of the study population, including child/adult categories (child/paediatric: 15 years and below; adult: above 15 years), gender, country of residence and malaria positivity.

From Table 1, a discrepancy between RDT (85.4\%) and microscopy (78.6\%) diagnosis was demonstrated. Out of the 88 participants who were $P$. falciparum positive by RDT, only 77 participants were $P$. falciparum positive by microscopy, rendering 11 from the RDT as

Table 1 Univariate analysis-demographic characteristics of patients attending selected health facilities in NorthWestern Province, Zambia enrolled into the study

\begin{tabular}{ll}
\hline Variables & Frequency $\mathbf{N}=\mathbf{1 0 3}$ \\
\hline Age category & \\
Child & $73(70.9 \%)$ \\
Adult & $30(29.1 \%)$ \\
Gender & \\
Female & $60(58.3 \%)$ \\
Male & $43(41.7 \%)$ \\
Country & \\
Zambia & $93(90.3 \%)$ \\
Congo \& Angola & $10(9.7 \%)$ \\
Rapid diagnostic test positivity & \\
Positive & $88(85.4 \%)$ \\
Negative & $15(14.6 \%)$ \\
Malaria microscopy positivity & \\
Positive & $81(78.6 \%)$ \\
Negative & $22(21.4 \%)$ \\
\hline
\end{tabular}


false positives. On the other hand, out of the 15 RDT negative participants, four were microscopically positive for other species. Figure 1 illustrates the enrolment process and sample characteristics.

Table 2 presents the three (3) variables used to describe the characteristics of malaria in the study as presence of (1) species, (2) gametocytes and (3) severe anaemia.

Overall, the 81 out of 103 patients that tested positive for malaria by microscopy translated to a prevalence of $78.6 \%$. The species found in the malaria positive participants were 76 (93.8\%) P. falciparum and 5 (6.2\%) for other species. Disaggregating the malaria cases by local and imported, $72(88.9 \%)$ of the 81 positive participants were local cases and $9(11.1 \%)$ were imported.
Based on the characteristics of both the local and imported malaria cases investigated, namely; species, gametocyte presence and severity of anaemia, this study shows that 71 of the 72 local cases (98.6\%) were $P$. falciparum and only one (1.4\%) was $P$. malariae; and five (three from Angola and two from Congo) out of nine imported cases (55.6\%) were P. falciparum although one case was a mixed infection of $P$. falciparum and $P$. malariae. The remaining four out of the nine imported cases (44.4\%) were of species other than $P$. falciparum broken down as follows: two out of nine P. ovale $(22.2 \%)$ occurring in participants from Angola and Congo each; one out of nine $(11.1 \%)$ with a mixed infection of $P$. ovale and P. malariae, occurring in a participant from Angola and yet another one out of nine

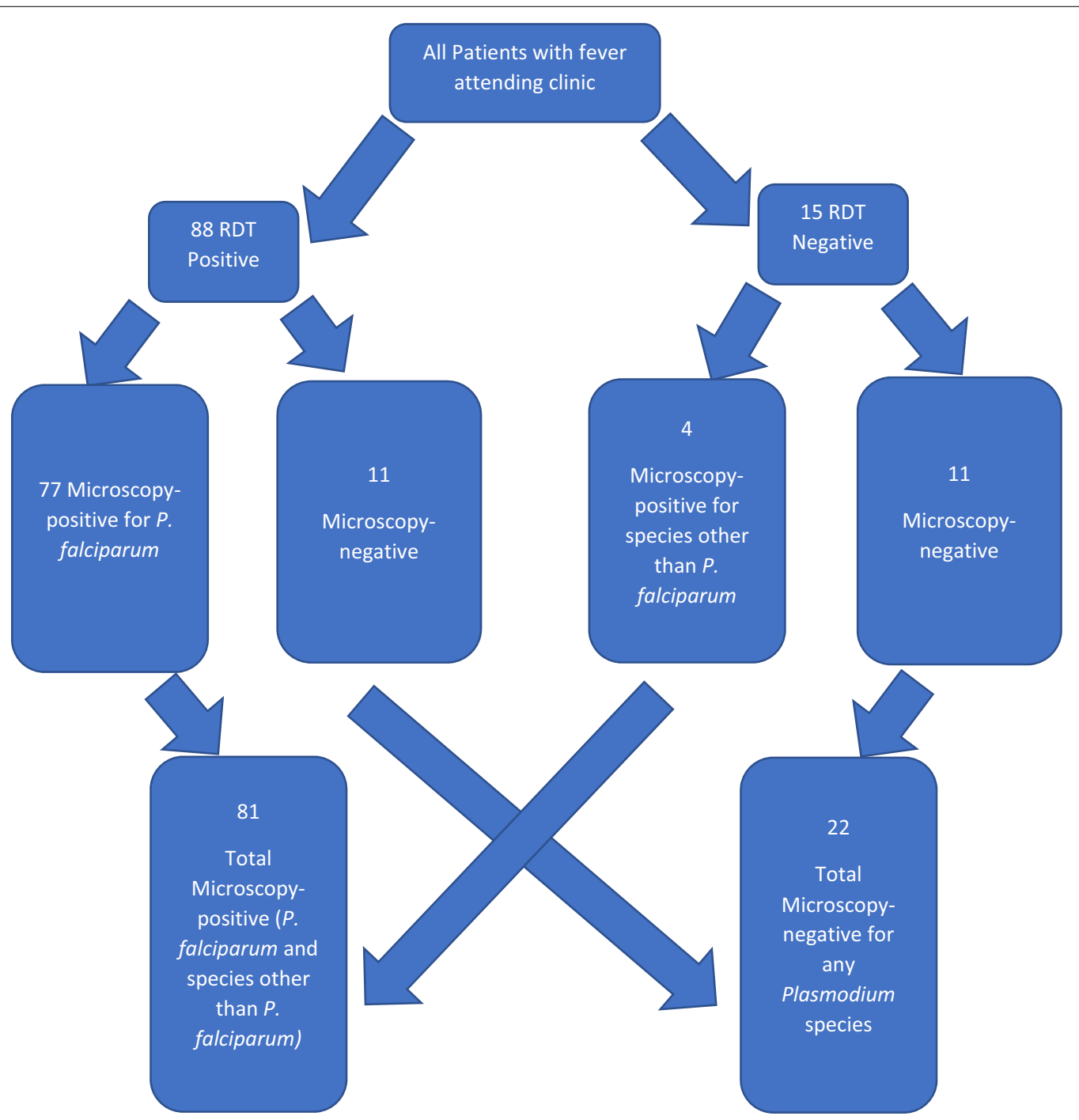

Fig. 1 Enrollment process for participants into the study 
Table 2 Bivariate analysis-risk factors for characteristics (species; gametocytes and severity) of local and imported malaria in patients attending selected health facilities in North-Western Province, Zambia enrolled into the study

\begin{tabular}{|c|c|c|c|c|c|c|}
\hline \multirow{3}{*}{$\begin{array}{l}\text { Independent } \\
\text { variables } \\
\text { Categories }\end{array}$} & \multicolumn{6}{|c|}{ Malaria characteristics (dependent variables) } \\
\hline & \multicolumn{2}{|l|}{ Species } & \multicolumn{2}{|l|}{ Gametocytes } & \multicolumn{2}{|l|}{ Anaemia } \\
\hline & $\begin{array}{l}1=\text { Species other } \\
\text { than P. falciparum }\end{array}$ & $0=P$. falciparum & $\begin{array}{l}1=\text { Gametocytes } \\
\text { present }\end{array}$ & $\begin{array}{l}0=\text { Gametocytes } \\
\text { absent }\end{array}$ & $1=$ Severe anaemia & $0=$ Moderate $/$ normal \\
\hline \multicolumn{7}{|l|}{ Age group } \\
\hline Children & $2(3.6 \%)^{*}$ & $53(96.4 \%)$ & $3(5.5 \%)$ & $52(94.5 \%)$ & $27(49.1 \%)$ & $28(50.9 \%)$ \\
\hline Adults & $3(11.5 \%)$ & $23(88.5 \%)$ & $3(11.5 \%)$ & $23(88.5 \%)$ & $11(42.3 \%)$ & $15(57.7 \%)$ \\
\hline \multicolumn{7}{|l|}{ Gender } \\
\hline Female & $3(6.1 \%)$ & $46(93.9 \%)$ & $4(8.2 \%)$ & $45(91.8 \%)$ & $26(53.1 \%)^{*}$ & $23(46.9 \%)$ \\
\hline Male & $2(6.3)$ & $30(93.7 \%)$ & $2(6.3 \%)$ & $30(93.8 \%)$ & $12(37.5 \%)$ & $20(62.5 \%)$ \\
\hline \multicolumn{7}{|l|}{ Country } \\
\hline $\begin{array}{l}\text { Participants from } \\
\text { Congo and } \\
\text { Angola }\end{array}$ & $4(44.4 \%)^{*}$ & $5(55.6 \%)$ & $4(44.4 \%)^{*}$ & $5(55.6 \%)$ & $3(33.3 \%)^{*}$ & $6(66.7 \%)$ \\
\hline $\begin{array}{l}\text { Participants from } \\
\text { Zambia }\end{array}$ & $1(1.4 \%)$ & $71(98.6 \%)$ & $2(2.8 \%)$ & 70 (97.2\%) & 35 (48.6\%) & $37(51.4 \%)$ \\
\hline
\end{tabular}

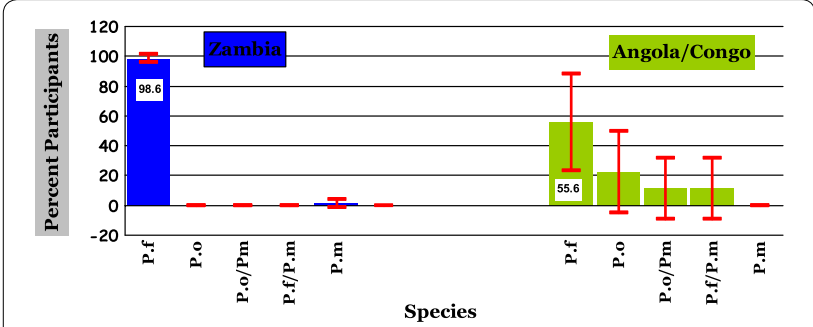

Fig. 2 Plasmodium species identified in the malaria cases recruited

(11.1\%) with a mixed infection of $P$. falciparum and $P$. malariae, occurring in a participant from Congo.

Figure 2 shows that the Plasmodium species picture in the participants resident in Zambia differs significantly from that in participants from the two neighbouring countries. Participants resident in Zambia had P. falciparum (98.6\%: 95\% CI 95.9-101.3) as the main species, while participants from the two neighbouring countries had a considerable combination of $P$. falciparum (55.6\%: 95\% CI 23.1-88), P. ovale (22.2\%: 95\% CI 4.9-49.4), P. ovale/P. malariae and P. falciparum/P. malariae (11.1\%: 95\% CI 9.4-11.1) each. Table 2 further shows that out of the five cases of species other than P. falciparum, three were adult cases (11.5\%) and two were children $\left(3.6 \% ; \chi^{2}=4.5 ; \mathrm{p}=0.03\right)$, varying significantly.

With regards to the other two characteristics studied, i.e. presence of gametocytes and severe anaemia, there were four participants (44\%) with gametocytes from the two neighbouring countries, which is significantly more than the two cases $\left(2.8 \% ; \chi^{2}=48 ; \mathrm{p}<0.001\right)$ observed in the participants resident in Zambia.

In terms of severe anaemia, Table 2 shows that this varied significantly by gender and country where 26 females (53.1\%) compared to 12 males representing $37.5 \%$ $\left(x^{2}=4.9 ; \mathrm{p}=0.03\right)$ and three $(33.3 \%)$ from the two neighbouring countries and $35(48.6 \%)$ from Zambia $\left(x^{2}=4.8\right.$; $\mathrm{p}=0.03)$ had severe anaemia.

Firth Penalized Regression models were run for the significant cases in the bivariate analysis to determine the associated risk factors. Table 3 shows that country of residence related positively to presence of species other than $P$. falciparum $(\mathrm{OR}=39.0, \mathrm{CI}[5.9,445.9]$; $\mathrm{P}<0.01)$ as well as presence of gametocytes $(\mathrm{OR}=23.1$, CI [4.2, 161.6]; $\mathrm{P}<0.01$ ). Thus participants who resided in Angola and Congo were more likely to have species other than $P$. falciparum as well as gametocytes. Age and gender were not associated with either presence of species other than P. falciparum or gametocytes. Country, age and gender were all not significant in the severe anaemia analysis.

These results show that: Predicted logit of (species presence) $=-3.864+(3.664) *$ Country; and Predicted logit of (gametocyte occurrence) $=-3.339+(3.139) *$ Country.

According to the models, the odds of patients residing in the two neighbouring countries who attend the selected health facilities in North-Western Province, having species other than P. falciparum were 39.1 times $\left(=\mathrm{e}^{3.664}\right.$; Table 3$)$ times greater than the odds of patients residing in Zambia who attend the same selected health facilities in North-Western Province having these species; and the odds of patients residing in the two 
Table 3 Firth (penalized) regression analysis of risk factors for characteristics of local and imported malaria in patients attending selected health facilities in North-Western Province, Zambia, enrolled into the study

\begin{tabular}{|c|c|c|c|c|c|c|c|c|}
\hline Predictor & Coef. & SE Coef. & Wald's $x^{2}$ & $d f$ & $p$ & $e \beta$ (odds ratio) & & \\
\hline \multicolumn{9}{|l|}{ Species } \\
\hline Constant & -3.864 & 0.831 & Inf & 1 & $<0.01$ & N/A & & \\
\hline Country ( 1 = Congo \& Angola; 0 =Zambia) & 3.664 & 1.067 & 14.7 & 1 & $<0.01$ & 39.0 & 5.9 & 445.9 \\
\hline Test overall model evaluation & & & $x^{2}$ & $d f$ & $p$ & & & \\
\hline Likelihood ratio test & & & 14.7 & 1 & $<0.01$ & & & \\
\hline Wald test & & & 11.8 & 1 & $<0.01$ & & & \\
\hline \multicolumn{9}{|l|}{ Gametocytes } \\
\hline Constant & -3.339 & 0.648 & $\operatorname{lnf}$ & 1 & $<0.01$ & N/A & & \\
\hline Country ( $1=$ Congo \& Angola; 0 =Zambia) & 3.139 & 0.932 & 12.5 & 1 & $<0.01$ & 23.1 & 4.2 & 161.6 \\
\hline Test overall model evaluation & & & $x^{2}$ & $d f$ & $p$ & & & \\
\hline Likelihood ratio test & & & 12.5 & 1 & $<0.01$ & & & \\
\hline Wald test & & & 11.3 & 1 & $<0.01$ & & & \\
\hline
\end{tabular}

Cl confidence interval, Coef. coefficient, SE coef. standard error of coefficient, $x^{2}$ Chi square; $d f$ degrees of freedom, $e \beta$ exponent of coefficient, Inf infinity

neighbouring countries who attend the selected health facilities in North-Western Province, having gametocytes were 23.1 times $\left(=\mathrm{e}^{3.139}\right.$; Table 3$)$ times greater than the odds of patients residing in Zambia who attend the same selected health facilities in North-Western Province, having gametocytes.

\section{Discussion}

This current study examines the characteristics of local and imported malaria cases in selected health facilities of some border areas of north-western Zambia. An overall malaria prevalence of $78.6 \%$ was observed. These findings are slightly higher but comparable with other findings [13] based on the analysis of data from the 2012 Zambia Malaria Indicator Survey (MIS) which showed $52.6 \%$ and $55.6 \%$ malaria prevalence for Eastern and Luapula provinces of Zambia respectively [13]. The findings are consistent with another study [7], which determined that the incidence of malaria in North-Western Province did not vary from that of Luapula Province. This was in the context that although North-Western Province belonged to the moderate malaria transmission zone in Zambia, its malaria burden approximated that of high malaria transmission zones [7]. As a matter of fact, north-western Zambia generally receives quite high rainfall amounts and has environmental conditions suitable for malaria vector breeding $[7,8]$. As such, malaria transmission zones are indicative of the general burden in delineated geographical locations based on general environmental and climatic conditions and yet actual burden may vary by specific places because the specific environmental and climatic conditions vary even within the same transmission zone [7].
Disaggregating the prevalence of malaria by local and imported cases, this study, demonstrated that majority $(88.9 \%)$ of the malaria cases were local, as expected, although a few (11.1\%) imported cases were also detected. These were of patients from the two neighbouring countries, Angola and Congo, who accessed the selected health facilities in Zambia during the period of our study. The two countries Angola and Congo have been reported to be among the leading countries in the importation of malaria from Africa to other countries, such as China [14]. With regards to imported malaria, this study discusses the findings in perspective of other national survey findings, in spite of the differences in study design and duration. Here this study employs the definition of imported malaria, that "cases are regarded as imported infections when in-migration occurred within 5-7 days of a case being detected" [32]. As such, the imported malaria prevalence in this study was lower than most surveys reviewed. The prevalence of imported malaria was higher in most studies; given the $64 \%$ in China [14], about 14 to $90 \%$ in Iran [33], 100\% in South Africa [34], 53\% in Swaziland [35] and 94.2\% in Lusaka, Zambia [36]. However, a study conducted in Botswana showed a lower prevalence of $6.01 \%$ [37], comparable to the findings in this study.

Discussing this study conducted in a moderate malaria transmission zone, in perspective these surveys, makes it clear that imported malaria occurs in low [34, 36], high [34] as well as moderate transmission zones, in varying proportions and without a pattern specific for particular zones. For a long time now the World Health Organization (WHO), has been recommending quantifying of the importation of infections into a region [38]. This is advised in order to classify regions according to 
their "vulnerability" [38], and also to estimate the risk of malaria being reintroduced following elimination efforts [39]. However, the focus has been more on the role of the latter, to prevent negating the malaria elimination successes [40, 41]. Given the possibility of malaria importation occurring in all zones of malaria transmission, it is very fundamental to pay attention to imported malaria infections even when local incidence is high [42]. This is crucial because importation will contribute to enhance the parasite reservoir and affect any elimination and control successes [42]. It has been recognized that staff at borders can also contribute to transmission of imported malaria [43], another reason why paying attention to imported malaria and border control is critical. Countries that have scored successes in eliminating malaria have among other strategies, employed border controls to detect and treat malaria infections before they reach receptive areas of the countries they enter [34]. Additionally, such countries have also integrated other strategies like economic development [32] and cross-border vector control [32, 40].

This study determined the characteristics of both the local and imported malaria cases based on species, gametocyte presence and severity of anaemia. The two models, show that country of residence is related to both species and gametocyte presence. P. falciparum as well as $P$. malariae and $P$. ovale, referred to as "species other than P. falciparum" in this study, were present in patients attending the selected health facilities of north-western Zambia. A prevalence of $93.8 \%$ P. falciparum and $6.2 \%$ of other Plasmodium species was found in this study. These findings are consistent with the Zambia 2012 MIS study, which demonstrated prevalence of $88.3 \%$ P. falciparum species and $11.6 \%$ non-falciparum species [13]. Like the current study, the MIS study [13] also demonstrated that Plasmodium species other than the main Plasmodium falciparum were present, especially in areas that have proximity to border areas [13]. The Zambia Ministry of Health publications also indicate that species other than Plasmodium falciparum are present in Zambia, in low proportions [28]. According to the Zambia Ministry of Health, Plasmodium falciparum accounts for more than 95\%, while $P$. malariae makes up $3 \%$ and $P$. ovale $2 \%$ [28].

In this study, the species other than $P$. falciparum, were missed by the initial RDT screening and were only detected by microscopy. This is attributed to the $P$. falciparum-specific RDTs employed. As a matter of fact, the P. falciparum-specific RDTs are the only kits available in most health facilities, as per recommendation and provision by the Ministry of Health [28]. This recommendation was effected on the acceptable premise of the country Zambia having over $95 \%$ of the malaria cases caused by one species [44]. However, the reality as demonstrated in this study, is that in the rare case when species other than P. falciparum are present, they will be missed and misdiagnosed $[28,45]$. It would be prudent for the government of Zambia to consider the use of RDTs that detect all species in health facilities located in border areas.

Apart from species, this study also assessed gametocyte presence in the blood smears collected and examined. Gametocytes are a natural phenomenon where malaria infections ultimately transition from asexual to sexual forms when in a human being [46-48] and are responsible for transmission of malaria from one host to another. This study compared the proportions of patients with gametocytes between the patients resident in the two neighbouring countries compared to those resident in Zambia. The proportions in the former were significantly higher than in the latter. The findings are interpreted with full recognition of the following considerations, that microscopy is limited in detecting gametocytes $[49,50]$; only a small fraction of asexual parasites differentiate into gametocytes [51] and only a fraction of infected individuals harbour gametocytes $[49,51]$. However, these results are acceptable given the efforts made to reduce bias in slide reading. The study involved up to three slide readers, all blinded to one another, for all the specimens. As such, the results are accurate to the extent of the capacity of the diagnostic tools employed. As others have suggested, it is important to acknowledge the role of gametocytes in malaria transmission [46-48]. This study further extrapolates this notion in perspective of its potential contribution to transmission of the non-falciparum species at the border areas of Zambia with the two neighbouring countries. All conditions permitting, this is a possibility as one study suggests that individuals who enter malaria regions can influence epidemiological dynamics [52]. It is necessary to study further the transmission potential that could exist with the presence of species other than $P$. falciparum and their gametocytes, as part of the process for planning and revising of control and elimination efforts.

With regards to severity of anaemia, the proportion of mild or moderate anaemia was higher than that of severe anaemia overall in our study. However, the cases of severe anaemia were higher in patients residing locally in Zambia than those residing in neighbouring countries. The fact that severe anaemia was not significant in the modelling is highlighted, although noting also that the proportions of severe anaemia were higher among patients with $P$. falciparum than among those infected with other species. This does not rule out the fact that severe anaemia still occurs with species other than $P$. falciparum $[2,28]$. This study underscores the fact that $P$. falciparum is virulent, and the heavy parasite burden associated with it lends to development of 
anaemia rapidly causing severe red cell haemolysis due to parasitaemia [53]. This could understandably justify the focus on P. falciparum malaria diagnosis in Zambia over the other species. With regards to other species, studies have shown that $P$. malariae is characterized by chronic infections, while $P$. ovale has dormant hypnozoites stages, which may explain the lower haemolysis rate [54]. In spite of the varying pathogenesis of malaria in infections with the different species, these infections still demand attention. This is explained in that they may each require additional and well considered administration of interventions, such as primaquine, an anti-hypnozoite drug [54] in the case of $P$. ovale. Severe anaemia cases were observed in both local participants as well as those from the two neighbouring countries, and in infections involving both $P$. falciparum and other species. It is, therefore, necessary for control programmes to consider studying the extents of anaemia severity that different species may be associated with so as to appropriately manage the infections. Severe anaemia is a recognized proxy for severe malaria and has been used to prioritize management of cases [26]. It is recommended that attention be paid to malaria severity with severe anaemia as a proxy, in the management of imported cases that tend to be more severe than local infections. Although this study determined that severity of malaria with regards to anaemia severity was higher in the local infections compared to the imported ones, the discussion is in perspective of other findings that imported malaria infections are more severe than local infections [55].

Age, gender and country of residence as risk factors for presence of particular malaria species and gametocytes were evaluated. However, only country of residence was significantly related. Although there were more females than males and more children than adults with malaria, these factors were not of significant relationship based on penalized logistic regression modelling. Notwithstanding, it is necessary for a country participating in malaria elimination programmes to consider exploring age and gender, as potential factors at play especially that these have been significant elsewhere [36]. The variation between genders has been shown to be possibly related to occupational exposure for women working in vending jobs at late hours [56, 57]. Others have further alluded to unequal balance of power between men and women in households, which bars women in households to access healthcare [57]. On the other hand, genetic factors have been advanced [58] and may be explored. Age as a factor remains critical as demonstrated in a similar study, which found that children were the most affected by imported malaria, advancing weaker immunity [36]. As such children should continue to be prioritized for treatment and care.

In this study, the country malaria patients resided in, was related to the malaria species they carried. While such information on species per country is available [2] and has served as guide on what species to focus on in control strategies among countries, these findings can also be used as an indicator of potential or active malaria importation.

This study was limited on the following scores: it was conducted outside the peak malaria transmission season in Zambia, which could have underestimated the cases detected; it excluded patients without signs and symptoms of malaria and thereby possibly missing asymptomatic cases; its screening capacity to detect all Plasmodium species by RDT was limited to $P$. falciparum specific tests, which could have been improved with RDTs that can detect all species; its capacity to confirm both species and gametocytes was limited to microscopy, which could have yielded a more accurate picture with more sensitive methods; it focussed only on malaria-infected humans and their travel history to confirm imported cases, when further tests to confirm onset of infection and detection of infection in vectors, could have increased accuracy.

\section{Conclusion}

Malaria prevalence in North-Western Province is high, with $P$. falciparum as the predominant species. Importation of P. ovale and P. malariae is happening in the province through human travel across borders with the two neighbouring countries. This study demonstrates that geographic residence of patients is a major risk factor for malaria species and gametocyte presence. It is relevant in that it highlights the status of local and imported malaria and risk factors that could be used for targeted response. The need for enhanced malaria control with specific focus on border controls to detect and treat, for specific diagnosis and treatment according to species obtaining, for further research in the role of species and gametocytes in imported malaria cannot be overemphasized.

\footnotetext{
Abbreviations

P: Probability value; $x^{2}$ : Chi-square test; OR: Odds ratio; Cl: Confidence interval; WHO: World Health Organization; spp: Species; NMEC: National Malaria Elimination Centre; E8: Elimination 8; Congo D. R.: Democratic Republic of Congo; RDTs: Rapid diagnostic tests; EDTA: Ethylene Diamine Tetra-acetic Acid; HB: Haemoglobin; pH: Potential of hydrogen; WBCs: White blood cells; Mm: Millimetres; G: Grams; DI: Decilitre; Coef:: Coefficient; SE coef:: Standard error of coefficient; Df: Degrees of freedom; EB: Exponent of coefficient; Inf: Infinity; SPSS: Statistical Package for Social Sciences; UNZAHSREC: University of Zambia Health Sciences Research Ethics Committee; NHRA: National Health Research Authority.
} 


\section{Acknowledgements}

We acknowledge the Ministry of Health Provincial Health office and the Health Centres for approval of the study and provisions towards it. We further acknowledge all participants.

\section{Authors' contributions}

MNC conceived the study and both authors contributed to the study design; NMS-M guided in the considerations for data collection and analysis; MNC collected the data; NMS-M guided the experiments and drafted the manuscript; both authors analysed the data and contributed to the interpretation and presentation of data. Both authors read and approved the final manuscript.

\section{Funding}

This work was self-funded by the two authors, with support in kind from the Health facilities and approval from Ministry of Health.

\section{Availability of data and materials}

The datasets used and/or analysed during the current study are available from the corresponding author on reasonable request.

\section{Ethics approval and consent to participate}

The study protocol (ID: 20181221003) was approved by University of Zambia Health Sciences Research Ethics Committee (UNZAHSREC) (IRB00011000 of IORG0009227) considering human participation. Confidentiality was observed and assurances given with regards to, drawing of blood, pain involved and age of certain participants. Informed consent process was duly conducted and consent obtained before participants were enrolled into the study. The National Health Research Authority (NHRA) approved the dissemination and publication of this study.

\section{Consent for publication}

Not applicable.

\section{Competing interests}

The authors as well as the funders declare that they have no competing interests in the manuscript.

Received: 17 June 2020 Accepted: 17 November 2020

Published online: 23 November 2020

\section{References}

1. Suh KN, Kain KC, Keystone JS. Malaria. Can Med Assoc J. 2004; 170:1693-702.

2. WHO. World malaria report. Geneva: World Health Organization; 2019.

3. USAID. President's malaria initiatives in Zambia. Zambia malaria operational plan. Fighting malaria and saving lives. Zambia profile. https:// www.pmi.gov/docs/default-source/default-document-library/malar ia-operational-plans/fy-2018/fy-2018-zambia-malaria-operational-plan. pdf?sfvrsn=7; 2018. Accessed 4 Sep 2020.

4. WHO. Roll Back Malaria. Progress and impact country reports: Focus on Zambia. Geneva: World Health Organization; 2011.

5. Kamuliwo M, Chanda E, Haque U, Mwanza-Ingwe M, Sikaala C, SakalaKatebe $\mathrm{C}$, et al. The changing burden of malaria and association with vector control interventions in Zambia using district-level surveillance data, 2006-2011. Malar J. 2013;12:437.

6. Masaninga F, Chanda E, Chanda-Kapata P, Hamainza B, Masendu HT, Kamuliwo $M$, et al. Review of the malaria epidemiology and trends in Zambia. Asian Pac J Trop Biomed. 2012;3:89-94

7. Shimaponda-Mataa NM, Tembo-Mwase E, Gebreslasie M, Achia TNO, Mukaratirwa S. Modelling the influence of temperature and rainfall on malaria incidence in four endemic provinces of Zambia using semiparametric Poisson regression. Acta Trop. 2017;166:81-91.

8. Kibret S, Lautze J, McCartney M, Nhamo L, Wilson GG. Malaria and large dams in sub-Saharan Africa: future impacts in a changing climate. Malar J. 2016;15:448.

9. Ministry of Health. Malaria Indicator Survey. Lusaka, 2016.

10. CDC. Where malaria occurs. https://www.cdc.gov/malaria/about/distr ibution.html. 2020. Accessed 4 Jun 2020
11. UNFPA. State of World Population. UNFPA Annual Report. New York: United Nations Population Fund; 2011.

12. Tatem AJ, Jia P, Ordanovich D, Falkner M, Huang Z, Howes R, et al. The geography of imported malaria to non-endemic countries: a meta-analysis of nationally reported statistics. Lancet Infect Dis. 2017:17:98-107.

13. Sitali L, Chipeta J, Miller JM, Moonga HB, Kumar N, Moss WJ, et al. Patterns of mixed Plasmodium species infections among children six years and under in selected malaria hyper-endemic communities of Zambia: population-based survey observations. BMC Infect Dis. 2015;15:204

14. Zhou R, Li S, Zhao Y, Yang C, Liu Y, Qian D, et al. Characterization of Plasmodium ovale spp imported from Africa to Henan Province, China. Sci Rep. 2019;9:2191.

15. Ministry of Health. National Malaria Elimination Strategic Plan 2017-2021. Lusaka, 2017.

16. Elimination 8. Zambia Country Overview. https://malariaelimination8. org/zambia/. 2020. Accessed 4 June 2020.

17. Sturrock HJW, Roberts KW, Wegbreit J, Ohrt C, Gosling RD. Tackling imported malaria: an elimination endgame. Am J Trop Med Hyg. 2015;93:139-44.

18. Freedman DO, Weld LH, Kozarsky PE, Fisk T, Robins R, von Sonnenburg $\mathrm{F}$, et al. Spectrum of disease and relation to place of exposure among ill returned travelers. N Engl J Med. 2006;354:119-30.

19. Muentener P, Schlagenhauf P, Steffen R. Imported malaria (1985 \pm 95 ): trends and perspectives. Bull World Health Organ. 1999;77:560-6.

20. Cao Y, Wang W, Liu Y, Cotter C, Zhou H, Zhu G, et al. The increasing importance of Plasmodium ovale and Plasmodium malariae in a malaria elimination setting: an observational study of imported cases in Jiangsu Province, China, 2011-2014. Malar J. 2016;15:459.

21. Central Statistical Office. 2010 Census of Population and Housing. Lusaka, 2012

22. Ministry of Health. Malaria Indicator Survey. Lusaka, 2010.

23. Shute GT. The microscopic diagnosis of malaria. In: Wernsdorfer WH, McGregor I, editors. Malaria, principles and practice of malariology. Edinburgh: Churchill Livingstone; 1988. p. 781-814.

24. Houwen B. Blood film preparation and staining procedures. Int J Lab Hematol. 2000:6:1-7.

25. WHO. Basic Malaria Microscopy. Geneva: World Health Organization; 2010. http://www.searo.who.int/LinkFiles/Malaria_malaria_microscopy _Learners_guide2010.pdf. Accessed 4 Sep 2020.

26. WHO. Guidelines for the treatment of malaria. 3rd ed. Geneva: World Health Organization; 2015.

27. Perkins DJ, Were T, Davenport GC, Kempaiah P, Hittner JB, Ong'echa JM. Severe malarial anemia: innate immunity and pathogenesis. Int J Biol Sci. 2011;7:1427-42.

28. Ministry of Health. Malaria Diagnosis and Treatment Guidelines. Lusaka, 2017.

29. King G, Zeng L. Logistic regression in rare events data. Polit Anal. 2001;9:137-63.

30. Heinze G, Schemper M. A solution to the problem of separation in logistic regression. Stat Med. 2002;21:2409-19.

31. Heinze G, Ploner M. Logistf: Firth's bias-reduced logistic regression. R package version 1.22. https://CRAN.Rproject.org/package=logistf; 2016. Accessed 27 Aug 2020.

32. Al Zahrani MH, Omar Al, Abdoon AMO, Ibrahim AA, Alhogail A, Elmubarak $M$, et al. Cross-border movement, economic development and malaria elimination in the Kingdom of Saudi Arabia. BMC Med. 2018;16:98.

33. Vatandoost H, Raeisi A, Saghafipour A, Nikpour F, Nejati J. Malaria situation in Iran: 2002-2017. Malar J. 2019:18:200.

34. Raman J, Gast L, Balawanth R, Tessema S, Brooke B, Maharaj R, et al. High levels of imported asymptomatic malaria but limited local transmission in KwaZulu-Natal, a South African malaria-endemic province nearing malaria elimination. Malar J. 2020:19:152.

35. Tejedor-Garavito N, Dlamini N, Pindolia D, Soble A, Ruktanonchai NW, Alegana $V$, et al. Travel patterns and demographic characteristics of malaria cases in Swaziland, 2010-2014. Malar J. 2017;16:359.

36. Lowa M, Sitali L, Siame M, Musonda P. Human mobility and factors associated with malaria importation in Lusaka district, Zambia: a descriptive cross sectional study. Malar J. 2018;17:404

37. Chihanga S, Haque U, Chanda E, Mosweunyane T, Moakofhi K, Jibril HB, et al. Malaria elimination in Botswana, 2012-2014: achievements and challenges. Parasit Vectors. 2016;9:99. 
38. Pampana E. Textbook of malaria eradication. 2nd ed. Oxford: Oxford University Press; 1969.

39. Cohen JM, Moonen B, Snow RW, Smith DL. How absolute is zero? An evaluation of historical and current definitions of malaria elimination. Malar J. 2010;9:213

40. Nikolov M, Bever CA, Upfill-Brown A, Hamainza B, Miller JM, Eckhoff PA, et al. Malaria elimination campaigns in the Lake Kariba Region of Zambia: a spatial dynamical model. PLoS Comput Biol. 2016;12:e1005192.

41. Porter TR, Finn TP, Silumbe K, Chalwe V, Hamainza B, Kooma E, et al. Recent travel history and Plasmodium falciparum malaria infection in a region of heterogenous transmission in Southern Province, Zambia. Am J Trop Med Hyg. 2020;103(Suppl 2):74-81.

42. Cohen JM, Le Menach A, Pothin E, Eisele TP, Gething PW, Eckhoff PA, et al. Mapping multiple components of malaria risk for improved targeting of elimination interventions. Malar J. 2017;16:459.

43. Cui L, Yan G, Sattabongkot J, Cao Y, Chen B, Chen X, et al. Malaria in the Greater Mekong Subregion: heterogeneity and complexity. Acta Trop. 2012;121:227-39.

44. Lee GC, Jeon ES, Le DT, Kim TS, Yoo JH, Kim HY, et al. Development and evaluation of a rapid diagnostic test for Plasmodium falciparum, P. vivax, and mixed-species malaria antigens. Am J Trop Med Hyg. 2011;85:989-93.

45. USAID. President's Malaria Initiatives in Zambia. Zambia Malaria Operational Plan. FY 2019. https://www.pmi.gov/docs/default-source/defaultdocument-library/malaria-operational-plans/fy19/fy-2019-zambia-malar ia-operational-plan.pdf?sfvrsn=3; 2019. Assessed 4 Sep 2020.

46. Josling GA, Llinas M. Sexual development in Plasmodium parasites: knowing when it's time to commit. Nat Rev Microbiol. 2015;13:573-87.

47. Talman AM, Domarle O, Mckenzie FE, Ariey F, Robert V. Gametocytogenesis: the puberty of Plasmodium falciparum. Malar J. 2004;3:24

48. Gardiner DL, Trenholme KR. Plasmodium falciparum gametocytes: playing hide and seek. Ann Transl Med. 2015;3:45.

49. Drakeley C, Sutherland C, Bousema JT, Sauerwein RW, Targett GAT. The epidemiology of Plasmodium falciparum gametocytes: weapons of mass dispersion. Trends Parasitol. 2006;22:424-30.
50. Babiker HA, Schneider P, Reece SE. Gametocytes: insights gained during a decade of molecular monitoring. Trends Parasitol. 2008;24:525-30.

51. Taylor LH, Read AF. Why so few transmission stages? Reproductive restraint by malaria parasites. Parasitol Today. 1997;13:135-40.

52. Thway AM, Rotejanaprasert C, Sattabongkot J, Lawawirojwong S, Thi A, Hlaing TM, et al. Bayesian spatiotemporal analysis of malaria infection along an international border: hlaingbwe Township in Myanmar and ThaSong-Yang District in Thailand. Malar J. 2018;17:428.

53. White NJ. Anaemia and malaria. Malar J. 2018:17:371.

54. Sitali L, Miller JM, Mulenga CM, Daniel JB, Moonga BH, Hamainza B, et al. Distribution of Plasmodium species and assessment of performance of diagnostic tools used during a malaria survey in southern and western provinces of Zambia. Malar J. 2019;18:130.

55. Dharmawardena P, Rodrigo C, Mendis K, Gunasekera WMTAW, Premaratne R, Ringwald P, et al. Response of imported malaria patients to antimalarial medicines in Sri Lanka following malaria elimination. PLoS ONE. 2017;12:e0188613.

56. Jenkins R, Omollo R, Ongecha M, Sifuna P, Othieno C, Ongeri L, et al. Prevalence of malaria parasites in adults and its determinants in malaria endemic area of Kisumu County. Kenya. Malar J. 2015;14:263.

57. WHO. Roll Back Malaria Global Technical Strategy for Malaria 2016-2030. Geneva: World Health Organization; 2015.

58. De Mendonca VRR, Goncalves MS, Barral-Netto M. The host genetic diversity in malaria infection. J Trop Med. 2012;2012:940616.

\section{Publisher's Note}

Springer Nature remains neutral with regard to jurisdictional claims in published maps and institutional affiliations.
Ready to submit your research? Choose BMC and benefit from:

- fast, convenient online submission

- thorough peer review by experienced researchers in your field

- rapid publication on acceptance

- support for research data, including large and complex data types

- gold Open Access which fosters wider collaboration and increased citations

- maximum visibility for your research: over $100 \mathrm{M}$ website views per year

At BMC, research is always in progress.

Learn more biomedcentral.com/submissions 